\title{
Alterations in Frequency Coding and Activity Dependence of Excitability in Cultured Neurons of Drosophila Memory Mutants
}

\author{
Ming-Li Zhao and Chun-Fang Wu \\ Department of Biological Sciences, University of lowa, lowa City, lowa 52242
}

Mutants of the Drosophila dunce (dnc) and rutabaga (rut) genes, which encode a cAMP-specific phosphodiesterase and a calcium/calmodulin-responsive adenylyl cyclase, respectively, are deficient in short-term memory. Altered synaptic plasticity has been demonstrated at neuromuscular junctions in these mutants, but little is known about how their central neurons are affected. We examined this problem by using the "giant" neuron culture, which offers a unique opportunity to analyze mutational effects on neuronal activity and the underlying ionic currents in Drosophila. On the basis of instantaneous frequency and first latency of spikes evoked by current steps, four categories of firing patterns (tonic, adaptive, delayed, and interrupted) were identified in wild-type neurons, revealing interesting parallels to those commonly observed in vertebrate CNS neurons. The distinct firing patterns were correlated with expression of different ratios of 4-aminopyridine- and tetraethylammonium- sensitive $\mathrm{K}^{+}$currents. Subsets of $d n c$ and rut neurons displayed abnormal spontaneous spikes and altered firing patterns. Altered frequency coding in mutant neurons was demonstrated further by using stimulation protocols involving conditioning with previous activity. Abnormal spike activity and reduced $\mathrm{K}^{+}$current remained in double-mutant neurons, suggesting that the opposite effects on cAMP metabolism by $d n c$ and rut do not counterbalance the mutual functional defects. The aberrant spontaneous activity and altered frequency coding in different stimulus paradigms may present problems in the stability and reliability of neural circuits for information processing during certain behavioral tasks, raising the possibility of modulation in neuronal excitability as a cellular mechanism underlying learning and memory.

Key words: dnc; rut; cAMP; spike activity; learning and memory; Drosophila giant neurons; firing patterns; potassium currents
Modification in synaptic strength and nerve terminal branching has been the focus in the study of cellular mechanisms underlying learning and memory (Bailey and Kandel, 1993; Bliss and Collingridge, 1993). cAMP-mediated signaling pathways have been associated with synaptic plasticity in many species, from synaptic facilitation in Aplysia (Kandel and Schwartz, 1982) to long-term potentiation in mammals (Frey et al., 1993; Huang et al., 1994; Weisskopf et al., 1994); however, cAMP-dependent modulation of ion channels (Levitan, 1988) has also been shown to modify impulse activities of neurons (Kaczmarek and Kauer, 1983). Because modulation of neuronal electrical properties can profoundly change the operation of neural networks (Getting, 1989; HarrisWarrick and Marder, 1991; Marcus and Carew, 1991), it may represent another important cellular mechanism for activitydependent conditioning of behavior.

In Drosophila, a combination of different approaches has established that $d n c$ and rut mutations affect learning behavior attributable to defects in cAMP metabolism (Dudai et al., 1976; Byers et al., 1981; Livingstone et al., 1984; Tully and Quinn, 1985; DeZazzo and Tully, 1995; Davis, 1996; Wustmann et al., 1996). Recently, these mutations have been demonstrated to alter synaptic transmission and nerve terminal arborization at larval neuromuscular junctions (Zhong and Wu, 1991; Zhong et al., 1992), to reduce growth cone motility in cultured larval CNS neurons

Received Oct. 28, 1996; revised Dec. 31, 1996; accepted Jan. 6, 1997.

This work was supported by National Institutes of Health Grants NS 26528 and HD 18577 to C.-F.W. We thank John Renger, Dr. Jeff E. Engel, and Dr. G. A Lnenicka for comments on a draft of this manuscript, and Peter Taft for technical assistance.

Correspondence should be addressed to Dr. Chun-Fang Wu, Department of Biological Sciences, University of Iowa, Iowa City, IA 52242.

Copyright (C) 1997 Society for Neuroscience $0270-6474 / 97 / 172187-13 \$ 05.00 / 0$
(Kim and Wu, 1996), and to disrupt habituation in an escape circuit of adult flies (Engel and Wu, 1996). Little is known, however, about how the electrical activities of central neurons are affected. Intracellular recording of action potentials has been performed on certain Drosophila neurons in vivo (Ikeda and Kaplan, 1974; Tanouye et al., 1981), but additional electrophysiological analyses have been limited by technical difficulties. The Drosophila "giant" neuron culture system has provided a preparation accessible to electrophysiological characterizations of neuronal activity and the underlying ionic currents (Wu et al., 1990; Saito and Wu, 1991, 1993; Zhao and Wu, 1994). These giant neurons are derived from cell division-arrested embryonic neuroblasts (Wu et al., 1990), which display different branching patterns and express various types of ion channels (Saito and Wu, 1991, 1993), transmitters (Huff et al., 1989), and neuron-specific antigens (Wu et al., 1990). Here we show that different categories of giant neurons exhibit distinct firing patterns, providing a basis for mutational analysis of neuronal activity.

Our results demonstrated altered firing patterns and aberrant spontaneous spikes in $d n c$ and rut neurons. Defects in spike frequency coding in mutant neurons were characterized under different stimulus protocols. Correlating current-clamp with voltage-clamp data in mutant cells suggested alterations in voltage-activated $\mathrm{K}^{+}$currents, which were shown to be involved in generating different firing patterns. The aberrant spontaneous activity, frequency coding, and modulation by previous conditioning in mutant neurons may affect the performance of the neural circuits that mediate different learning behaviors. The neuronal defects in learning mutants $d n c$ and rut support the notion that modulation of excitability may serve as a potential cellular mechanism for learning and memory. 
Preliminary results have been published previously in abstract form (Zhao and Wu, 1994).

\section{MATERIALS AND METHODS}

Drosophila stocks. The wild-type strain Canton-Special, homozygotes of two different alleles of $d n c$ ( $\left(n c^{1}, y d n c^{2} e c f\right)$, two alleles of rut $\left(r u t^{1}, y\right.$ $r u t^{2}$ ), and the double-mutant strain $y d n c^{M 11} c v v r^{2} t^{2} f$ were raised on standard Drosophila medium at room temperature.

Cell culture. Cultures of Drosophila "giant" neurons, derived from cytokinesis-arrested embryonic neuroblasts, were prepared as described previously (Wu et al., 1990; Saito and Wu, 1991). Briefly, female flies were allowed to lay eggs on agar plates for 1-2 hr. The embryos that were collected were incubated for $3-4 \mathrm{hr}$ at $25^{\circ} \mathrm{C}$ and then homogenized in modified Schneider medium (Life Technologies, Gaithersburg, MD) containing $200 \mathrm{ng} / \mathrm{ml}$ insulin (Sigma, St. Louis, MO), 20\% fetal bovine serum (FBS), $50 \mu \mathrm{g} / \mathrm{ml}$ streptomycin, and $50 \mathrm{U} / \mathrm{ml}$ penicillin (all from Life Technologies). Cells were collected after centrifugation and resuspended in medium containing $1-2 \mu \mathrm{g} / \mathrm{ml}$ cytochalasin B (Sigma). Cells were plated on uncoated glass coverslips, and cultures were maintained in humidified chambers at room temperature $\left(21-24^{\circ} \mathrm{C}\right)$. Cytochalasin B was removed either by replacing with fresh culture medium a few hours after plating or by washing with physiological saline (see below) before recording. No evident differences in physiological results were noticed between the two treatments (cf. Saito and Wu, 1991; Zhao et al., 1995).

Electrophysiological recordings and data analysis. Whole-cell patchclamp recording has been described previously (Saito and Wu, 1991; Zhao et al., 1995). Patch electrodes were pulled from glass capillaries (75 $\mathrm{ml}$ micropipettes, VWR, Chicago, IL) and had a tip resistance of 2-4 M $\Omega$ when measured in the recording solution. Whole-cell recordings were obtained primarily from monopolar or bipolar neurons (soma diameter $\sim 15 \mu \mathrm{m}$ ) by using an EPC-7 patch-clamp amplifier (Medical Systems, Greenvale, NY). The seal resistance was usually $>8 \mathrm{G} \Omega$, and the junction potential was nulled just before rupture of the cell membrane. Bath solution contained $128 \mathrm{~mm} \mathrm{NaCl}, 2 \mathrm{~mm} \mathrm{KCl}, 4 \mathrm{~mm} \mathrm{MgCl}, 1.8 \mathrm{~mm} \mathrm{CaCl}_{2}$, and $35.5 \mathrm{~mm}$ sucrose, buffered at pH 7.1-7.2 with 5 mM HEPES (adjusted with $\mathrm{NaOH}$ ). Patch pipettes were filled with a solution containing $144 \mathrm{~mm}$ $\mathrm{KCl}, 1.0 \mathrm{mM} \mathrm{MgCl}_{2}, 0.5 \mathrm{mM} \mathrm{CaCl}_{2}$, and $5.0 \mathrm{~mm}$ EGTA, buffered at $\mathrm{pH}$ 7.1-7.2 with $10 \mathrm{mM}$ HEPES (adjusted with $\mathrm{KOH}$ ). An IBM-compatible computer equipped with A/D, D/A converters (Labmaster, Axon Instruments, Foster City, CA) was used with pClamp software (Version 5.51, Axon Instruments) for voltage/current pulse generation and data acquisition. Data were digitized at $1-5 \mathrm{kHz}$. All figures were constructed using software Clampfit (pClamp 5.51, Axon Instruments), Sigmaplot (Jandel Scientific, San Rafael, CA), and Superpaint (version 2.0 for Macintosh, Silicon Beach, San Diego, CA). Cell membrane capacitance $(C)$ was determined by the following equation: $C=Q / V$, under voltage-clamp conditions. Briefly, a small depolarization pulse, $V(5 \mathrm{mV})$, was delivered from a holding potential of $-80 \mathrm{mV}$. $Q$ was the integrated, total charge measured by using pClamp software.

Functional categorization of cell types. The firing patterns of individual neurons were determined by step current injections of $600 \mathrm{msec}$ at an intensity of two- to threefold threshold level. Firing patterns were categorized in wild-type neurons according to the following criteria involving latency to the first spike (latency) and instantaneous firing frequency determined by the reciprocal of interspike intervals. In particular, the intervals between the first two spikes $\left(f_{\text {first }}\right)$ and between the last two spikes $\left(f_{\text {last }}\right)$ in the spike train were used for distinguishing four different firing patterns. (1) Delayed: the onset of spikes showed a significant delay with a latency $>100 \mathrm{msec}$. (2) Adaptive: these neurons were normally conformed to the criteria of a latency $<100 \mathrm{msec}$ and $f_{\text {last }} / f_{\text {first }}<0.7$. They displayed decreasing firing frequency within a spike train. (3) Tonic: the latency was $<100 \mathrm{msec}$ and $f_{\text {last }} / f_{\text {first }}>0.7$. The firing frequency in these neurons was relatively constant and did not show a general trend of decrease. (4) Interrupted: the latency for the first spikes was $<100 \mathrm{msec}$, but there was a quiescent period of interruption before the reinitiation of a spike train with a sustained, high firing frequency, such that $f_{\text {last }} / f_{\text {first }}>$ 2.5 . The critical values of latency and the $f_{\text {last }} / f_{\text {first }}$ ratio for the above categorization were chosen because they described clear functional categories with minimal overlaps in all of the wild-type neurons obtained for this study. Except for some restricted cases (shown in Fig. 7 and discussed in Results), the same set of criteria has been applied to the analysis of the firing patterns of all-or-none spikes in mutant neurons. (The above criteria could not be applied to a few strongly adaptive cells in which only one or two spikes were generated during the $600 \mathrm{msec}$ period. These spikes occurred with a latency $<100 \mathrm{msec}$, and a spike train was not

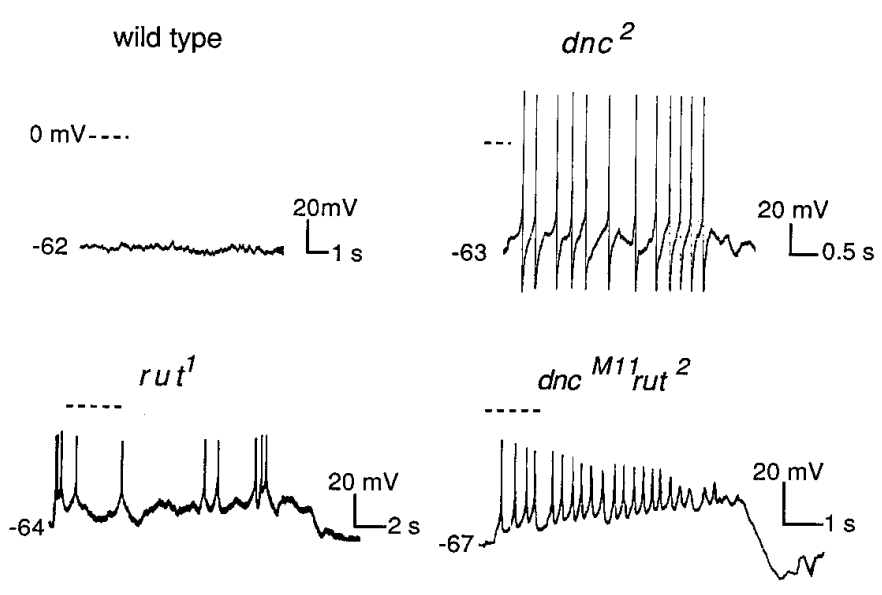

Figure 1. Spontaneous firing in subsets of mutant neurons. Examples show regenerative spikes in the absence of current injection in isolated "giant" neurons from $d n c^{2}, r u t^{1}$, and $d n c^{M 11} r u t^{2}$ cultures. Long-lasting plateau potentials were seen in rut and $d n c$ rut but not $d n c$ cultures. The resting potential and the zero (dashed lines) membrane potential are indicated. Note differing time scales.

evoked with increased stimulus intensity. Such cells were not included in Figs. 6, 9, and 10, but were reported only in Tables 1 and 2.)

\section{RESULTS}

Whole-cell current-clamp and voltage-clamp records were obtained mainly from monopolar and bipolar neurons (soma size 13-18 $\mu \mathrm{m}$ ). Homozygous mutants of two dunce alleles, $d n c^{1}, d n c^{2}$, two rutabaga alleles, $r^{1}{ }^{1}$, rut ${ }^{2}$, and double mutant $d n c^{M 11}{ }^{1} u t^{2}$ were examined in this study, whereas Canton-Special wild-type flies were used as controls. Three types of membrane potential changes in response to step current injections, all-or-none, graded, and nonregenerative were observed in wild-type (Saito and $\mathrm{Wu}, 1991$ ) and mutant neurons (Zhao and $\mathrm{Wu}, 1994)$. The distributions of these three types of cells were similar among cultures of different genotypes and were consistent with a previous report on wild-type cultures (Saito and Wu, 1991). The three types of neuronal excitability were also observed in dissociated embryonic neurons grown in a modified culture medium (O'Dowd, 1995). We found that the mutant phenotypes were most evident in the firing patterns observed in cells exhibiting all-or-none action potentials, which constituted a major population in different genotypes (wild type, 153/300; $d n c^{1}, 28 / 50 ; d n c^{2}, 73 / 137 ;$ rut $^{1}, 124 /$ 220; rut ${ }^{2}, 43 / 89 ;$ dnc $^{M 11}$ rut $\left.^{2}, 93 / 163\right)$.

\section{Abnormal spontaneous firing in mutant neurons}

An immediate hallmark of the mutant cultures was the spontaneous firing activities observed in subsets of neurons (Fig. 1). In contrast, wild-type neurons remained quiescent unless step current stimulation was applied $\left(d n c^{2}, 9 / 73 ;\right.$ rut $^{1}, 19 / 124$; and $d n c^{M 11} r u t^{2}, 15 / 93$; vs wild type, 0/153). Figure 1 shows examples of repetitive overshooting impulses in a $d n c$ neuron that lasted for seconds and sporadic oscillations seen in a rut neuron. It should be noted that nonovershooting spontaneous spikes were more prevalent in rut, whereas both overshooting and nonovershooting impulses were frequently encountered in $d n c$ neurons. In addition, long-lasting plateau potentials occurred in rut (not shown) and dnc rut neurons (Fig. 1), in which spikes of decreasing amplitude turned into sustained depolarization. These observations imply that abnormal neuronal hyperexcitability is caused by both 

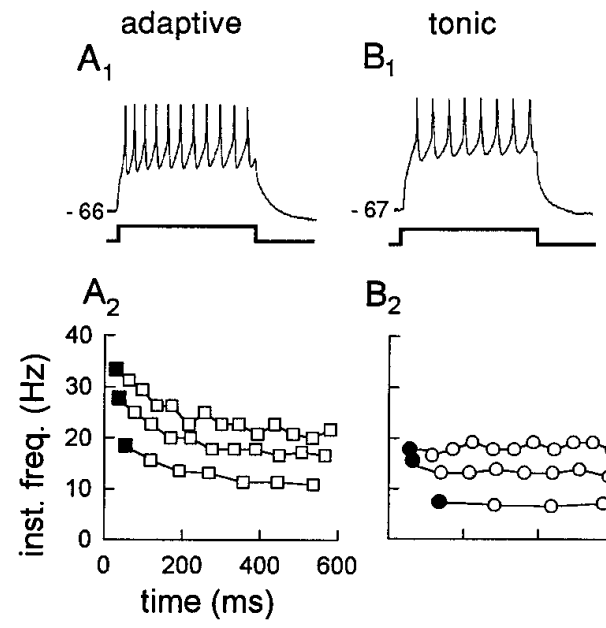

$\mathrm{B}_{2}$
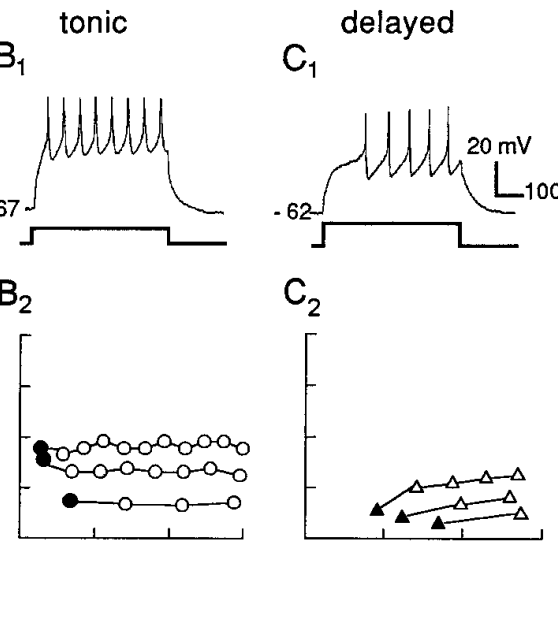

$d n c$ and rut mutations, despite their opposite effects on cAMP metabolism.

Other functional abnormalities were present in the mutant neurons, as indicated by the aberrant firing patterns induced by different paradigms of current injection. To analyze the disrupted mechanisms, a functional categorization of the cell types in the wild-type cultures must be established to allow an investigation on how cells of different categories are affected by $d n c$ and rut mutations.

\section{Classification of firing patterns in wild-type neurons}

A diversity of firing patterns in response to constant current injections is a hallmark of neurons in the CNS in many invertebrates and vertebrates (Byrne, 1980; Getting, 1983; Connors and Gutnick, 1990; Kawaguchi, 1995). Similarly, a diverse profile of firing was observed in giant neuron cultures of Drosophila. Among cells showing all-or-none action potentials in response to step current injections, four categories of firing patterns, distinct in their instantaneous frequency and first spike latency, were characterized in wild-type neurons (for criteria, see Materials and Methods). Figure 2 shows examples of the four firing patterns: adaptive $\left(A_{1}\right)$, tonic $\left(B_{1}\right)$, delayed $\left(C_{1}\right)$, and interrupted $\left(D_{1}\right)$. The instantaneous frequencies after the onset of step current injection in four representative cells were plotted at three stimulus intensities (Fig. $2 A_{2}-D_{2}$ ). The solid symbols indicate the occurrence of the first spikes and the reciprocal of the first spike latency. It is worth noting that the categories of firing patterns in Drosophila neurons reported here have also been described in neurons of many species, including mammals (Llinás, 1988; Connors and Gutnick, 1990).

Because neurons showing interrupted firing patterns were rarely encountered ( $<5 \%$ overall), they were excluded from further analysis. The remaining three major categories of wild-type neurons exhibited distinct electrical properties and pharmacological differences that are illustrated below and summarized in Table 1. This provides additional distinctions among neurons of different functional categories and their alterations in mutant cultures.

\section{Voltage-dependent $\mathrm{K}^{+}$currents in the regulation of firing patterns}

In identified neurons of other species, prepulse protocols have been shown to be an effective means for detecting the presence of a transient, inactivating $\mathrm{K}^{+}$current and assessing its involvement in regulating spiking activities (Byrne, 1980; Getting, 1983). As
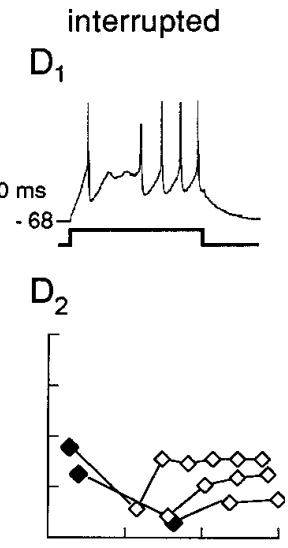

Figure 2. Categories of firing patterns of all-ornone action potentials in wild-type neurons. $A_{1}-$ $D_{1}$, Examples of whole-cell recordings from four individual neurons exhibiting adaptive, tonic, delayed, and interrupted firing patterns. Open symbols in $A_{2}-D_{2}$ plot the instantaneous frequency of spikes against time (after the onset of stimulation) with three current intensities (same data from cells in $A_{1}-D_{1}$ ). Filled symbols in $A_{2}-D_{2}$ signify the latency of the first spikes evoked by step current injections.

shown in Figure $3 A$, spike initiation by current injection was modified differentially by conditioning prepulses in different categories of neurons. Either a hyperpolarizing or a subthreshold current pulse preceding a suprathreshold depolarization was used to modify spike trains in adaptive (left), tonic (middle), and delayed (right) wild-type neurons. In delayed neurons, the onset of spikes was greatly facilitated by subthreshold depolarizing prepulses and retarded by hyperpolarizing prepulses. Interestingly, the opposite was true in adaptive cells under an identical paradigm. Furthermore, a small gradual depolarization ("hump") was frequently induced by a subthreshold stimulus in adaptive cells (Fig. 3A, Table 1). In general, tonic neurons displayed an intermediate sensitivity to modification by prepulses.

The diverse firing patterns may reflect differences in the expression of different ion channels among distinct neuron types. Voltage-dependent $\mathrm{K}^{+}$currents have been shown to be crucial in the regulation of neuronal firing patterns in many species (Hille, 1992). In Drosophila, voltage-activated $\mathrm{K}^{+}$currents have been characterized extensively in muscle fibers (Salkoff, 1983; Wu and Haugland, 1985; Singh and Wu, 1989; Wu and Ganetzky, 1992; Wang and Wu, 1996) and in cultured neurons (Byerly and Leung, 1988; Solc and Aldrich, 1988; Baker and Salkoff, 1990; Saito and Wu, 1991, 1993; O’Dowd, 1995; Zhao et al., 1995). In particular, the feasibility of correlating neuronal spike activities with the underlying ionic currents in the giant neuron culture system allowed us to examine the roles of these currents in the regulation of firing patterns of isolated single neurons.

We found evidence for differential expression of 4-aminopyridine (4-AP)-sensitive (transient) and tetraethylammonium (TEA)sensitive (delayed) $\mathrm{K}^{+}$currents in different cell types. Bath application of 4-AP (1 or $2 \mathrm{~mm}$ ) increased the firing frequency and shortened the latency to the onset of spikes to a different extent in each cell type (Fig. 3B). Clearly, these changes were most pronounced in delayed cells (right) and least evident in adaptive cells (left). In sharp contrast, adaptive cells were the most sensitive, and delayed neurons the most resistant, to modification by TEA (Fig. 3C). Bath application of TEA ( 5 or $10 \mathrm{~mm}$ ) broadened the duration of action potentials, reduced the rate of spike repolarization, and changed the shape of afterhyperpolarization most significantly in adaptive cells (left).

We directly correlated current- and voltage-clamp data in the same cells to further investigate the involvement of different types of voltage-dependent $\mathrm{K}^{+}$currents in distinct firing patterns. The left column in Figure 4 shows trains of spikes evoked in adaptive $\left(A_{1}\right)$, tonic $\left(B_{1}\right)$, and delayed $\left(C_{1}\right)$ wild-type neurons. After identification 


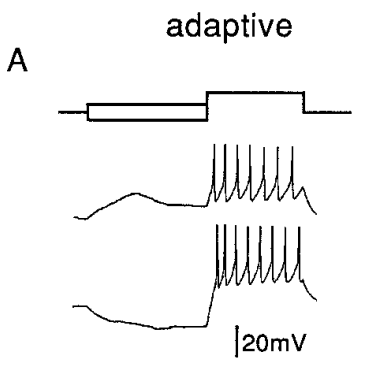

B

Figure 3. Roles of $\mathrm{K}^{+}$currents in the regulation of neuronal firing in wild-type cultures. $A$, Delayed cells appeared to be most sensitive to modification by preconditioning pulses. Trains of spikes from adaptive (left), tonic (middle), and delayed (right) neurons were elicited by suprathreshold current injections preceded by either a hyperpolarization (bottom trace) or a subthreshold depolarization (top trace). B, Delayed cells were most sensitive to 4-AP treatment. Spikes of adaptive (left), tonic (middle), and delayed (right) neurons before (control, top) and after (bottom trace) 4-AP treatment were compared. $C$, Adaptive cells were most sensitive to modification by TEA. Action potentials of adaptive (left), tonic (middle), and delayed (right) neurons before (control, top) and after (bottom trace) TEA treatment were compared.
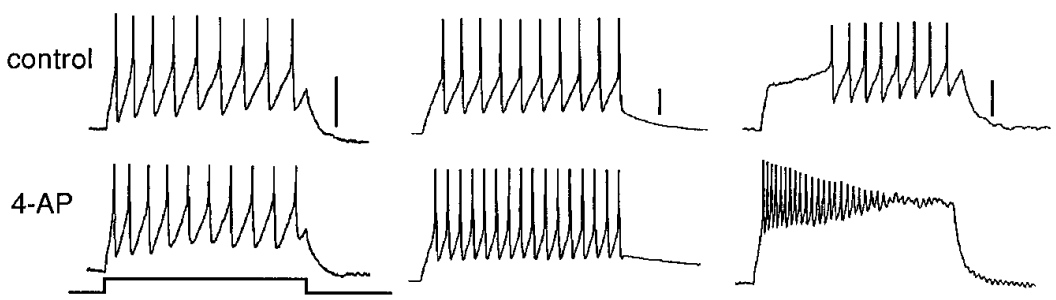

C
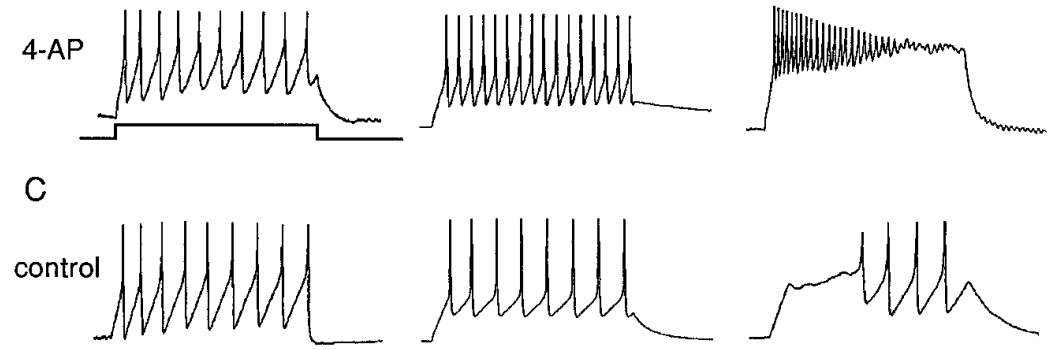

TEA
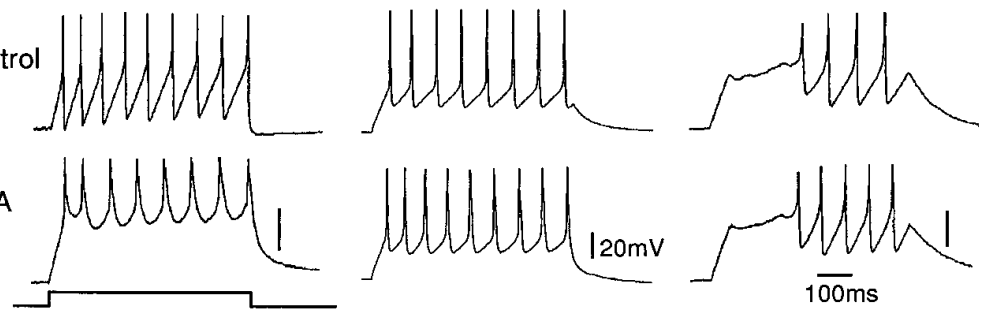

of the firing patterns under the current-clamp condition, tetrodotoxin (TTX) and $\mathrm{Cd}^{2+}$ were applied to the bath to eliminate voltageactivated $\mathrm{Na}^{+}$and $\mathrm{Ca}^{2+}$, and $\mathrm{Na}^{+}$- and $\mathrm{Ca}^{2+}$-dependent $\mathrm{K}^{+}$currents (Saito and $\mathrm{Wu}, 1991,1993)$. The voltage-activated outward $\mathrm{K}^{+}$ currents were isolated in this manner from adaptive $\left(A_{1}\right)$, tonic $\left(B_{1}\right)$, and delayed $\left(C_{1}\right)$ neurons and characterized under voltage-clamp conditions. Delayed cells seemed to express more fast-activating, transient $\mathrm{K}^{+}$currents, especially in the lower voltage range (Fig. $4 C_{2}$ ). In comparison, $\mathrm{K}^{+}$currents in adaptive cells activated more slowly and showed much less inactivation (Fig. $4 A_{2}$ ).

Table 1. Electrical properties of wild-type neurons exhibiting different firing patterns

\begin{tabular}{|c|c|c|c|}
\hline & Tonic & Adaptive & Delayed \\
\hline Input resistance $(\mathrm{M} \Omega)^{a}$ & $1316 \pm 91(45)$ & $1238 \pm 116(21)$ & $1052 \pm 162(15)$ \\
\hline Resting potentials $(\mathrm{mV})^{b}$ & $-63.6 \pm 1.1(49)$ & $-59.6 \pm 1.5(32)^{*}$ & $-54.5 \pm 2.0(14)^{* *}$ \\
\hline Threshold $(\mathrm{mV})^{c}$ & $-35.7 \pm 2.6(16)$ & $-38.5 \pm 2.0(35)$ & $-26.1 \pm 5.0(8)^{*}$ \\
\hline Spike amplitude $(\mathrm{mV})^{d}$ & $58.6 \pm 3.8(28)$ & $56.8 \pm 2.7(28)$ & $57.8 \pm 7.9(6)$ \\
\hline Duration $(\mathrm{msec})^{e}$ & $2.9 \pm 0.4(33)$ & $2.9 \pm 0.4(19)$ & $1.9 \pm 0.2(8)$ \\
\hline Response to subthreshold stimulation ${ }^{f}$ & No & Yes & No \\
\hline Oscillation before spikes ${ }^{g}$ & No & No & Yes \\
\hline Anodal break ${ }^{h}$ & Yes & Yes & No \\
\hline Modification by prepulse ${ }^{i}$ & ++ & + & +++ \\
\hline$I_{\mathrm{K}} / I_{A}^{j}$ & $\simeq 1$ & $>2$ & $\simeq 0.3$ \\
\hline
\end{tabular}

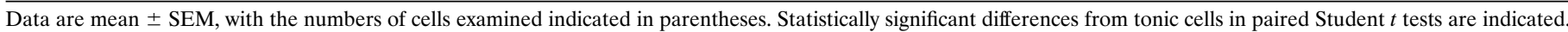
${ }^{*} p<0.05 ;{ }^{* *} p<0.01$.

${ }^{a}$ Input resistance: measured as the membrane voltage change from the resting potential divided by the amplitude of the hyperpolarizing current step applied.

${ }^{b}$ Resting potentials were measured immediately after forming the whole-cell configuration.

${ }^{c}$ Threshold for action potentials: determined as the take-off (inflection point) membrane potential of the first spike in response to step current injections.

${ }^{d}$ Spike amplitude: voltage difference between the resting potential and the peak of the first action potential.

${ }^{e}$ Duration: half-width of the first action potential, determined as the time between the peak and threshold.

${ }^{f}$ In response to subthreshold current injections, adaptive cells often showed an aborted regenerative hump (Fig. $3 \mathrm{~A}$, top trace).

${ }^{g}$ In response to suprathreshold current injections, delayed neurons oscillate before the onset of spikes (Fig. $3 C$ ).

${ }^{h}$ Spike initiation after release from strong hyperpolarizing current.

${ }^{i}$ The sensitivity of neurons to preconditioning by subthreshold depolarization and hyperpolarization as indicated by changes in the delay of spike onset (see Fig. $3 A$ ).

${ }^{j} I_{\mathrm{K}} / I_{\mathrm{A}}$ indicate here as the ratio of TEA-sensitive $\left(I_{\mathrm{K}}\right)$ and 4-AP-sensitive $\left(I_{\mathrm{A}}\right)$ potassium current components (see Fig. 4). 
A. adaptive

$$
\text { A1 }
$$

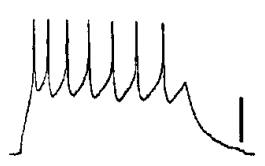

B. tonic

B1

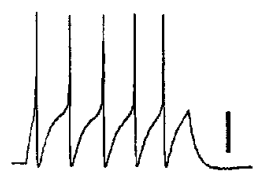

A2

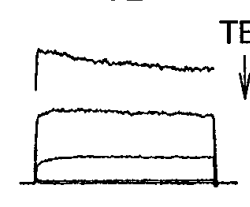

A3

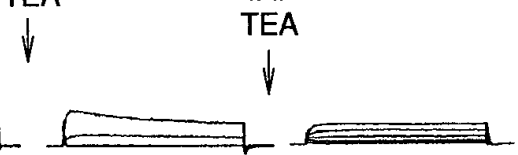

TEA sensitive 4-AP sensitive

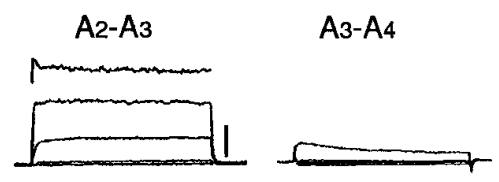

C. delayed

$\mathrm{C}_{1}$

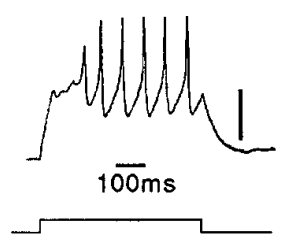

$\mathrm{B} 2$

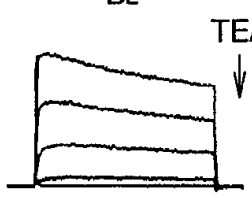

TEA

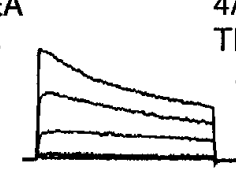

B4

$4 \mathrm{AP}+$

TEA

$\checkmark$
$\mathrm{B} 2-\mathrm{B} 3$

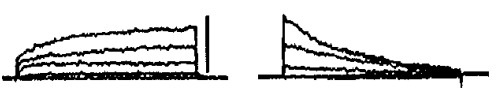

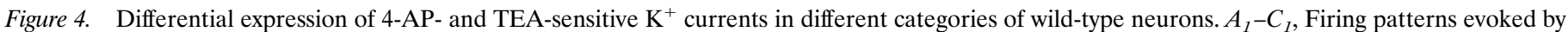

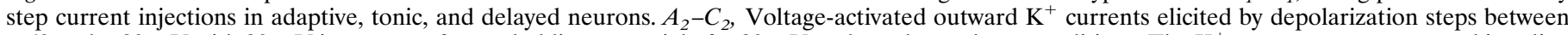

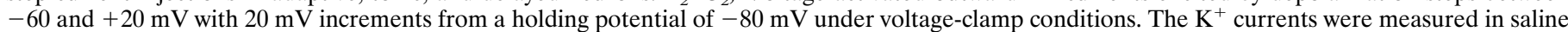

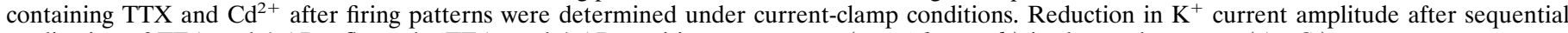
application of TEA and 4-AP reflects the TEA- and 4-AP-sensitive components (two right panels) in the total currents $\left(A_{2}-C_{2}\right)$.

4-AP (1 mM) and TEA (5 mM) were sequentially applied to examine the relative abundance of transient and slow inactivating components in cells displaying different firing patterns (Fig. 4). The 4-AP- and TEA-sensitive $\mathrm{K}^{+}$currents were then isolated by subtracting the remaining $\mathrm{K}^{+}$currents after each treatment from the currents before the treatment. Consistent with the above current-clamp results (Fig. 3), TEA-sensitive, slowly inactivating $\mathrm{K}^{+}$currents were predominant in adaptive cells, whereas 4-APsensitive, transient $\mathrm{K}^{+}$currents were prevalent in delayed cells (Fig. 4, right panels; also see Table 1). Tonic cells showed an intermediate amplitude for both TEA- and 4-AP-sensitive components (Fig. 4, right panels; Table 1). It should be noted that the proportion of the remaining 4-AP- and TEA-insensitive $\mathrm{K}^{+}$currents (Fig. $4 A_{4}-C_{4}$ ) varied in neurons and was not an indicator for different neuron types.

Taken together, these results show that the adaptive, tonic, and delayed firing patterns arise, at least in part, because of the differential interplay of distinct voltage-activated $\mathrm{K}^{+}$currents with inward $\mathrm{Na}^{+}$and $\mathrm{Ca}^{2+}$ currents. For example, the delayed onset type of firing most likely results from the inhibitory but selfinactivating effects of transient $\mathrm{K}^{+}$currents (Byrne, 1980; Getting, 1983; Rogawski, 1985); however, $\mathrm{Ca}^{2+}$ - and $\mathrm{Na}^{+}$-dependent $\mathrm{K}^{+}$ currents may also play some roles in determining the firing pattern in Drosophila neurons (Saito and Wu, 1991, 1993). The roles of these currents require further investigation.

\section{Altered frequency coding in mutants}

The above analyses provide a mechanistic basis and lend strong support for the validity of the categories of firing patterns proposed in Figure 2. We applied the same criteria (see Materials and
Methods) for categorization of firing patterns to mutant neurons exhibiting all-or-none action potentials to examine how different cell types were disrupted by $d n c$ and rut mutations. We often observed irregular spike activities in substantial fractions of neurons in mutant cultures (Table 2; Figs. 5, 6). In addition, a variety of extreme hyperexcitability was seen in mutant neurons whose firing patterns and erratic waveforms suggest different mechanisms in spike generation (Fig. 7 and Table 2, erratic firing). They were excluded from the four categories described above.

Figure 5 shows examples of altered spike activities observed from mutant neurons exhibiting adaptive, tonic, and delayed firing patterns. Brief bursting activity during current injection and longlasting plateau potentials after the cessation of stimuli were the hallmarks of mutant neurons.

Figure 6 presents instantaneous frequency plots of neurons during suprathreshold stimulation (two to three times the threshold level) (see Materials and Methods) for adaptive $(A)$, tonic $(B)$, and delayed $(C)$ types in wild-type, $d n c^{2}, r u t^{1}$, and $d n c^{M 11} r u t^{2}$ neurons. Several conclusions could be drawn on the basis of a large sample size. First, subsets of mutant neurons lacked fidelity in frequency coding attributable to fluctuations in firing rate. Wild-type controls varied to some degree in firing rate but followed a general pattern of frequency coding over time for each type of firing. In contrast, subsets of mutant cells displayed abrupt bursting activities and greater variation in firing rate. It seemed that more severe abnormalities occurred more often in tonic and delayed than in adaptive mutant neurons, indicating possible differential effects of the cAMP cascade in different cell types. Second, long-lasting plateau potentials were observed in mutant 
Table 2. Distribution of cells exhibiting distinct firing patterns

\begin{tabular}{|c|c|c|c|c|c|c|c|}
\hline \multirow[b]{2}{*}{ Genotype } & \multicolumn{4}{|c|}{ Firing patterns ( $\%$ of cells) } & \multirow{2}{*}{$\begin{array}{l}\text { Total } \\
\text { number of } \\
\text { cells }^{a}\end{array}$} & \multirow{2}{*}{$\begin{array}{l}\text { Spontaneous } \\
\text { spikes }^{b} \\
\text { (number of cells) }\end{array}$} & \multirow{2}{*}{$\begin{array}{l}\text { Erratic firing }{ }^{c} \\
\text { (number of cells) }\end{array}$} \\
\hline & Tonic & Adaptive & Delayed & Interrupted & & & \\
\hline Wild type & 45 & 32 & 19 & 4 & 152 & 0 & 0 \\
\hline$d n c^{1}$ and $d n c^{2}$ & 39 & 23 & 35 & 3 & 28 and 72 & 9 & 2 \\
\hline$r u t^{1}$ and $r u t^{2}$ & 44 & 27 & 26 & 3 & 114 and 43 & 19 & 4 \\
\hline$d n c^{M 11} r u t^{2}$ & 42 & 27 & 31 & 0 & 93 & 15 & 8 \\
\hline
\end{tabular}

${ }^{a}$ Total number of cells includes neurons displaying all-or-none action potentials and identified within the four firing patterns.

${ }^{b}$ Spike trains in the absence of current injections in some mutant neurons (compare Fig. 1) that are included in the "total number of cells" shown in the column.

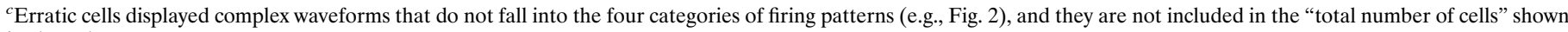
in the column.

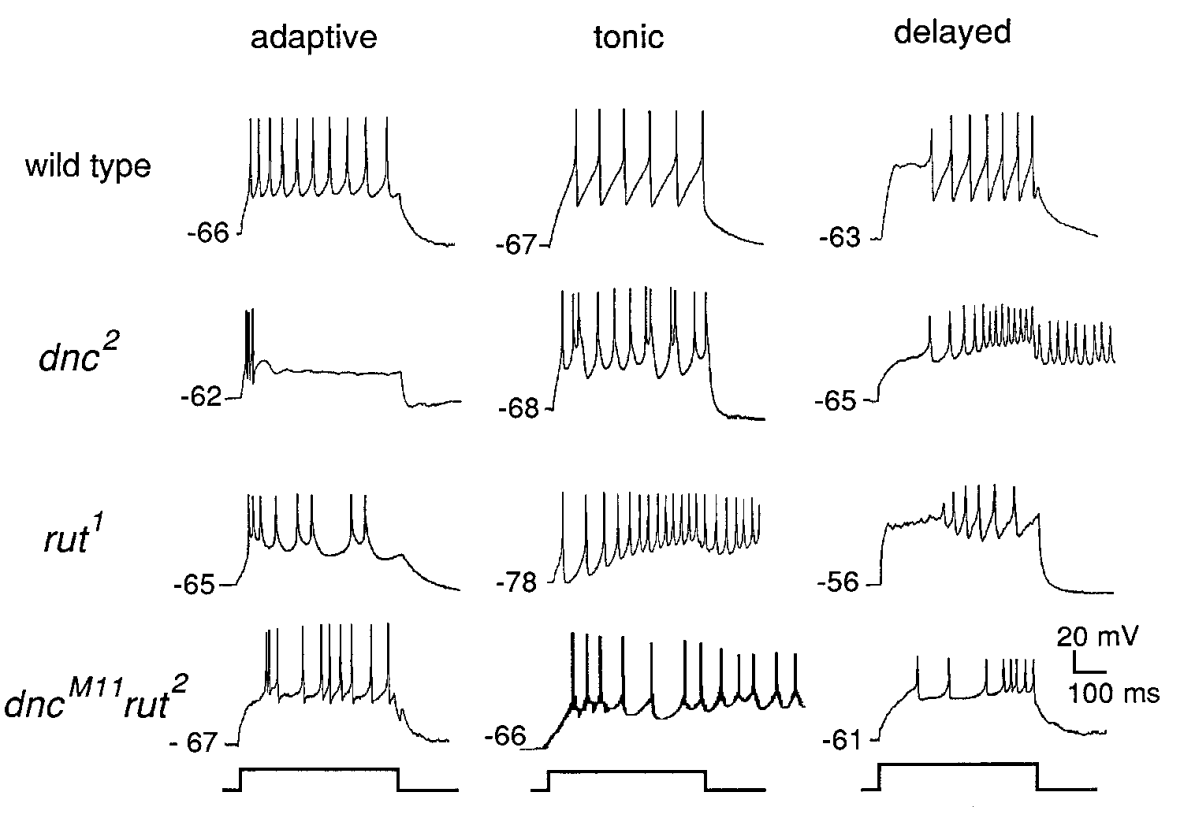

Figure 5. Examples of irregularities observed in dnc, rut, and dnc rut neurons displaying adaptive, tonic, and delayed firing patterns. Note the brief bursting and long-lasting plateau potentials. The resting potential is indicated.

neurons (Figs. 5, 6). These cells fired continuously at a relatively stable rate even after the cessation of current injection (marked by arrows in Figure 6). This type of abnormal firing seemed to be more abundant in $r u t^{1}$ than $d n c^{2}$ or $d n c^{M 11} r u t^{2}$. Finally, aberrant firing was found in the double-mutant $d n c^{M 11} r u t^{2}$, suggesting that $d n c$ does not functionally rescue the rut defect, consistent with the poor performance of the double mutants in learning behavioral tests (Livingstone et al., 1984).

As mentioned above, in a subpopulation of isolated mutant neurons, depolarizing current pulses frequently evoked strikingly aberrant electrical activities (Table 2, erratic firing), which do not fall into the above four firing categories. Examples are shown in Figure 7. A large proportion of these cells displayed spikes of small amplitudes and high firing rate, and prolonged plateau potentials associated with small amplitude, high-frequency oscillations (Fig. 7). Although the exact mechanisms are not known, the extreme excitability patterns in these isolated single cells might arise from distinct hyperexcitable sites on neurites, or they might be initiated through interactions among different cellular regions such as autapses (Bekkers and Stevens, 1991).

\section{Altered spike activities after a conditioning stimulus in mutants}

The functional categories of different cell types with distinct firing patterns led us to try additional stimulation paradigms to reveal further defects in mutant neurons. Two stimulation paradigms were found to be effective in characterizing frequency coding under activity-dependent conditioning. A ramp stimulus can be used to indicate the rate of accommodation and a paired-pulse protocol can reveal the influence of previous activity. Figure 8 shows an example of the results from a wild-type (Fig. $8 A_{1}$ ) and a dnc (Fig. $8 B_{1}$ ) neuron. In response to a suprathreshold step current injection, both tonic neurons exhibited similar firing rates (Fig. $8 C_{1}$ ). Nevertheless, clear changes of activities were observed in the $d n c$ neuron in subsequent tests with ramp (Fig. $8 B_{2}, C_{2}$ ) and double-pulse (Fig. $8 B_{3}, C_{3}$ ) stimuli. In response to a ramp depolarization, the wild-type neuron generated a train of impulses with gradually increased firing rate, which was silenced by the termination of depolarization (Fig. $8 A_{2}, C_{2}$ ). In contrast, the $d n c$ neuron showed irregularities in firing rate and an increase in spike instantaneous frequency, reaching a maximum of $50 \mathrm{~Hz}$, followed by a long-lasting train of spikes after the cessation of the ramp (Fig. $8 B_{2}, C_{2}$ ). In response to a twin-pulse depolarization, unlike the wild-type neuron, the $d n c$ neuron displayed abnormal facilitation, with more spikes evoked by the test pulse than by the conditioning pulse (Fig. 8). These observations suggested that more subtle mutational effects on different firing patterns could be revealed using these stimulation paradigms.

Ramp stimulation was systematically applied to examine mutational effects on neuronal accommodation to previous depolarization (Fig. $8 A_{2}, B_{2}$ ). In Figure 9, examples of spikes evoked by a 3 

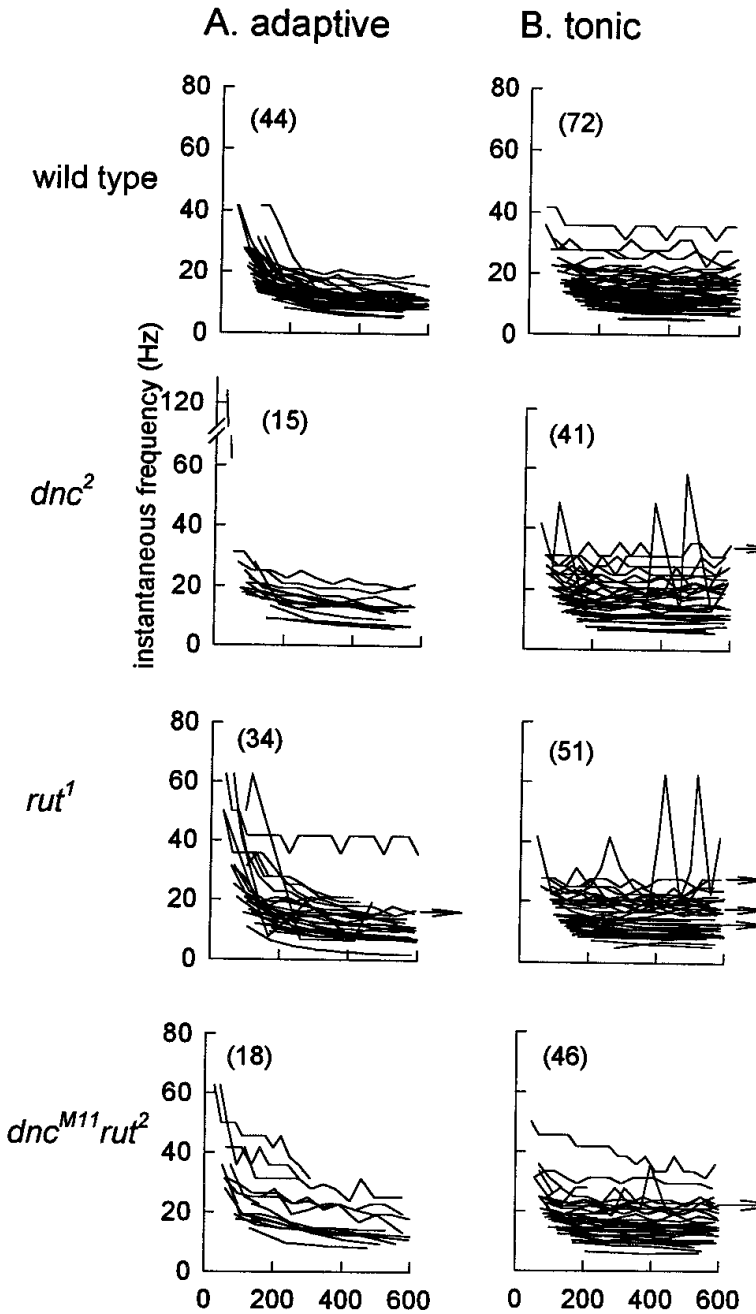
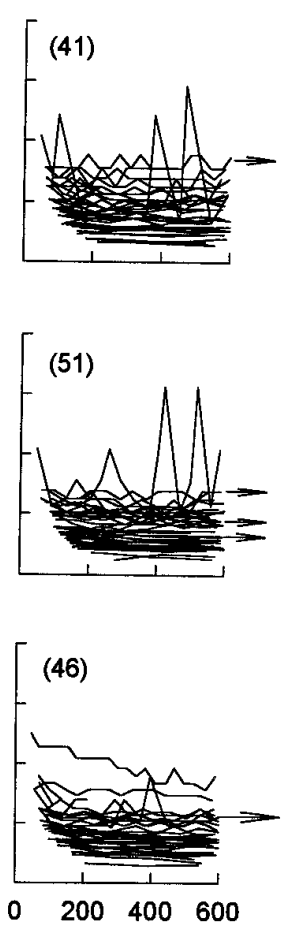

C. delayed
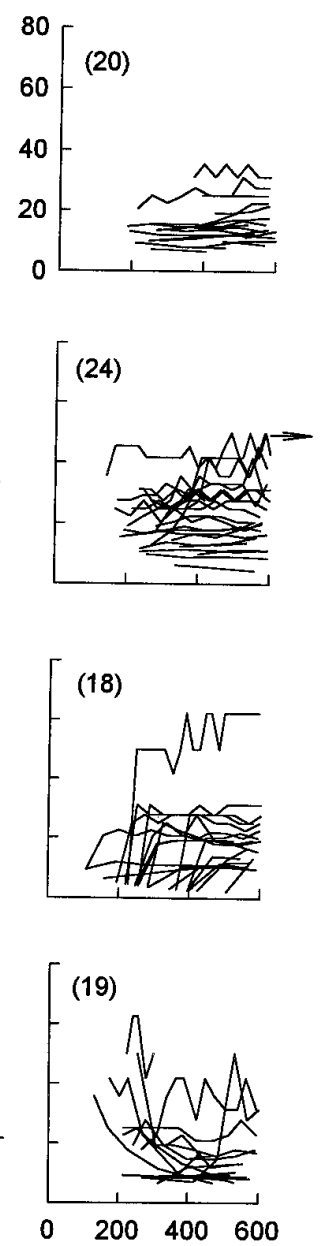

Figure 6. Instantaneous firing frequency of spike trains evoked by current injections in adaptive $(A)$, tonic $(B)$, and delayed $(C)$ neurons of wild-type and mutants, $d n c$, $r u t$, and $d n c$ rut. Segments in each continuous line connect the data points representing the instantaneous frequency of successive spikes for each neuron (calculated from the reciprocal of interpulse intervals; see Materials and Methods). Arrows indicate long-lasting firing after the cessation of current stimulation $(600 \mathrm{msec})$. The number of cells for each genotype is shown in the parentheses. For the sake of clarity, information about the first spike (reciprocal of the latency, see Fig. 2) in each neuron was omitted in the plots. sec ramp stimulation in adaptive $(A)$, tonic $(B)$, and delayed $(C)$ neurons of wild type (top) and different mutants (bottom) are shown. The instantaneous firing frequency was measured and plotted against time during ramp stimulation for a quantitative comparison (Fig. 9D-F).

In general, wild-type neurons in all three categories displayed a gradual increment in firing rate during ramp stimulation. Various altered spike activities in mutant neurons was revealed by ramp depolarization. First, long-lasting firing was maintained in subsets of mutant neurons after the cessation of a ramp stimulus $\left(d n c^{2}\right.$, 3/48; rut ${ }^{1}, 2 / 42 ; d n c^{M 11}{ } u t^{2}, 1 / 31$ ) (Fig. 9B,D-F), which lasted for several seconds in some cases. In contrast, only brief afterpotentials occurred occasionally in wild-type neurons. Second, fluctuations in firing frequency and striking bursting activity were evident in mutant neurons (Fig. 9A,E,F). Notably, bursts of spikes were not only evoked by strong depolarization but also occurred frequently in the early phase of stimulation, presumably a nearthreshold effect in mutant neurons. Third, compared with step current injections, $d n c$ mutations showed more profound defects with a ramp stimulus, especially in delayed and tonic neurons, whereas rut mutations appeared less abnormal during a ramp.

Abnormalities in response to previous activity conditioning were demonstrated further in mutant neurons by the paired-pulse paradigms. Figure $10 A, B$ illustrates spike trains from an adaptive $r u t^{1}$ and a delayed $d n c^{2}$ neuron evoked by the conditioning (left panel) and test (right panel) pulses in comparison to wild-type controls. Data obtained from neurons of different genotypes were quantified by plotting the cumulative change in number of spikes ( $n_{\text {test }}-n_{\text {cond. }}$ ) after the onset of the test pulse, i.e., the difference in the number of spikes accumulated over stimulus duration between the test and conditioning pulses. The resultant plots for tonic (Fig. $10 C$ ), adaptive (Fig. 10D), and delayed (Fig. 10E) neurons of wild type and mutants are shown. We found that wild-type tonic neurons exhibited relatively little sensitivity to prepulse conditioning, with only small cumulative changes in spike number (Fig. $10 C)$. Adaptive cells often showed less firing after the preconditioning pulse (Fig. $10 D ;\left(n_{\text {test }}-n_{\text {cond }}<0\right)$, whereas delayed neurons showed an increased cumulative change of spike numbers (Fig. $10 E$; a positive trend of accumulation, $n_{\text {test }}-n_{\text {cond }}>0$ ).

It is clear that neurons in mutant cultures displayed a much greater range of variation, with either enhancement or decrement of spike numbers, in response to test pulses after preconditioning stimulation (Fig. 10). Furthermore, the trends in cumulative changes observed in the adaptive and delayed wild-type cells were no longer evident. For example, in both single and double mutants, a portion of delayed neurons showed a reduction, instead of an increase, of spikes after preconditioning (Fig. 10E). These observations illustrate that firing activity became less predictable in mutant neurons.

The results obtained by applying the ramp and twin-pulse stimulus paradigms further elucidated modulation of neuronal activities by previous activity, which might be important to the 

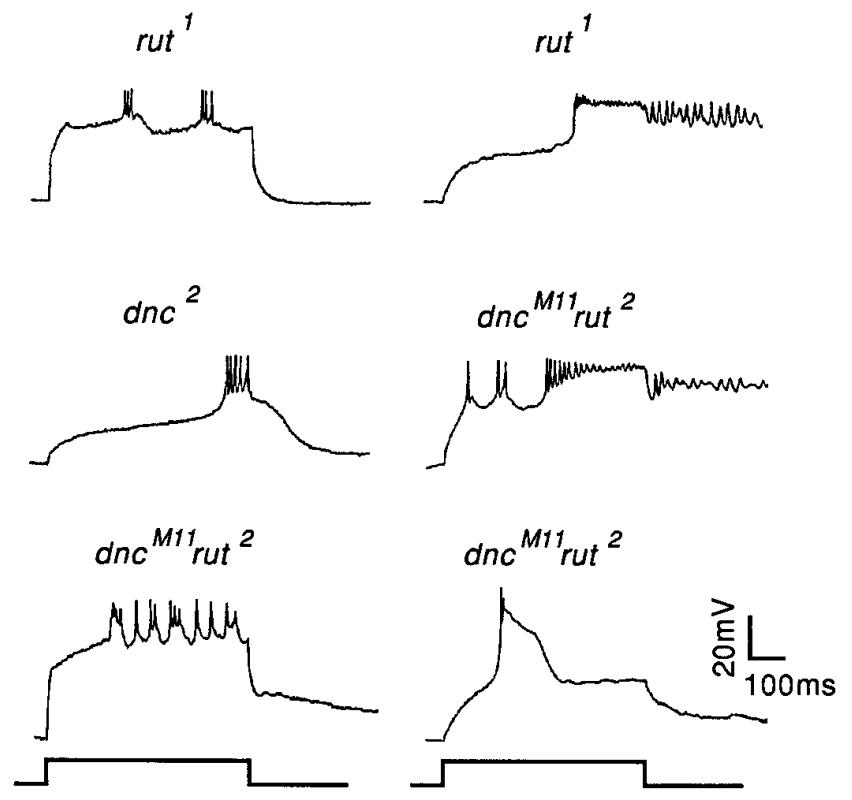

Figure 7. Highly irregular, abnormal electrical activities observed in isolated mutant neurons. Such complex waveforms do not fall into the four categories of firing patterns (cf. Materials and Methods, and Fig. 2). They were not seen in isolated wild-type neurons and could not be attributable to synaptic interactions between neurons. Note bursts of spikes, longlasting plateau potentials, and the small amplitude of action potentials evoked by 600 msec step current injections.

experience-dependent modification of behavior in flies. It seemed that such processes are altered in subsets of $d n c$ and rut neurons. Differential severity was observed, depending on categories of firing patterns and on specific stimulation paradigms.

\section{Reduced density of voltage-dependent $\mathrm{K}^{+}$currents in rut and dnc rut}

To investigate the ionic mechanisms underlying the altered electrical activities observed in mutants, pharmacological experiments were first carried out to test whether certain $\mathrm{K}^{+}$channel blockers could phenocopy the mutant defects in wild-type neurons. As shown above, voltage-activated $\mathrm{K}^{+}$channels may play major roles in the regulation of firing patterns in Drosophila neurons. Strikingly, wild-type tonic neurons showed long-lasting plateau firing after treatment by both $2 \mathrm{~mm}$ 4-AP and $10 \mathrm{~mm}$ TEA (Fig. 11A). This pattern of gradual damping of spikes into an oscillatory plateau also occurred spontaneously (data not shown). The robust firing pattern mimicked the extreme phenotype seen in neurons of rut $^{1}$ (Fig. 11A) and $d n c^{M 11}$ rut $^{2}$ (data not shown).

The above observation suggested defects in voltage-gated $\mathrm{K}^{+}$ currents in these mutants. This was supported by direct voltageclamp measurements of the underlying $\mathrm{K}^{+}$currents in $r u t^{1}$ neurons that had been shown to display long-lasting plateau potentials (Fig. $11 \mathrm{~B})$, and by voltage-clamp data pooled from populations of mutant neurons without correlation to current-clamp data (Fig. $11 C$ ). In comparison with the wild-type control (Fig. 11C, circles), the density of both transient (Fig. 11C, left) and steady-state (Fig. $11 C$, right $) \mathrm{K}^{+}$currents was significantly reduced in rut and dnc rut neurons. It should be noted, however, that other ion currents in addition to $\mathrm{K}^{+}$currents may be altered in mutant neurons. It is well known that cAMP can modulate different inward and outward currents (Levitan, 1988; Li et al., 1992). In addition, we found that the effects of $d n c$ mutations on $\mathrm{K}^{+}$currents were more

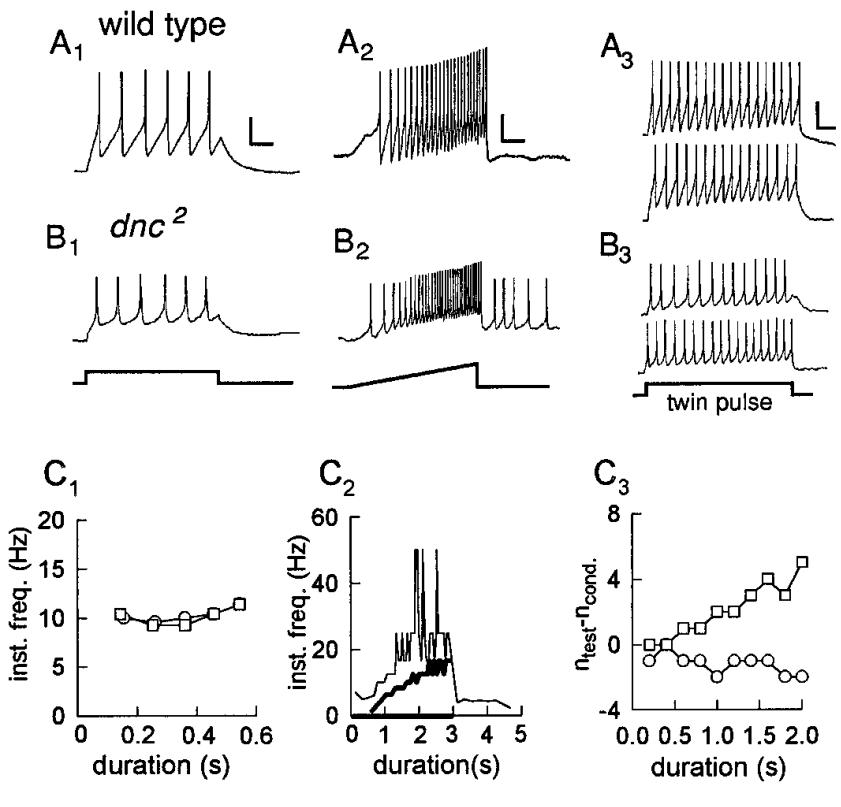

Figure 8. Patterns of electrical activities of a wild-type and a $d n c^{2}$ neuron subjected to different current injection paradigms. $A_{1}-A_{3}$, Patterns of spike activity in a "tonic" wild-type neuron elicited by step $\left(A_{1}, 600 \mathrm{msec}\right)$, ramp $\left(A_{2}, 3 \mathrm{sec}\right)$, twin pulses $\left(A_{3}, 2 \mathrm{sec}\right.$; conditioning, top; test, bottom $)$ from $B_{1}-B_{3}$. The same paradigms applied to a "tonic" $d n c^{2}$ mutant neuron. $C_{1}, C_{2}$, Plots of instantaneous frequency for spike trains evoked by step current injection and ramp stimulation. $C_{3}$, Cumulative differences of the number of spikes between conditioning and test pulses (see text). Calibrations: vertical, $20 \mathrm{mV}$ for all panels; horizontal, $100 \mathrm{msec}\left(A_{1}, B_{1}\right)$, $500 \mathrm{msec}\left(A_{2}, B_{2}\right), 300 \mathrm{msec}\left(A_{3}, B_{3}\right)$. Wild type, circles in $C_{1}$ and $C_{3}$ and the thin line in $C_{2} ; d n c$, squares in $C_{1}$ and $C_{3}$ and the thick line in $C_{2}$. Note that clear abnormalities in the $d n c$ neuron, which appeared close to normal in response to step current injection, were revealed by the ramp and twin-pulse paradigms.

complex, including a change in the voltage-dependence of channel activation, which would require more detailed analysis involving additional voltage-clamp paradigms and will be reported elsewhere.

\section{DISCUSSION}

This study shows for the first time that cultured Drosophila embryonic neurons can be categorized functionally according to their distinct firing patterns and differential expression of $\mathrm{K}^{+}$currents of different kinetic and pharmacological properties. These studies lay a foundation for the electrophysiological characterization of functional alterations of central neurons from Drosophila mutants of interest. Our results suggest that frequency coding and activitydependent plasticity of excitability are altered in neurons of $d n c$ and rut memory mutants, which are defective in the cAMP cascade. It is therefore proposed that modulation of neuronal excitability by second messenger cascades may serve as an important cellular mechanism underlying learning and memory.

\section{Ion channels, neuronal activity, and neural plasticity: role of the cAMP cascade}

The giant neuron cultures of Drosophila contain various cell types differentiated from cell division-arrested neuroblasts. Previous studies have demonstrated that these multinucleated cells express neuron-specific antigens and preserve morphological and functional diversity (Wu et al., 1990; Saito and Wu, 1991, 1993; Zhao and $\mathrm{Wu}, 1994)$. Using this culture system, the present study 

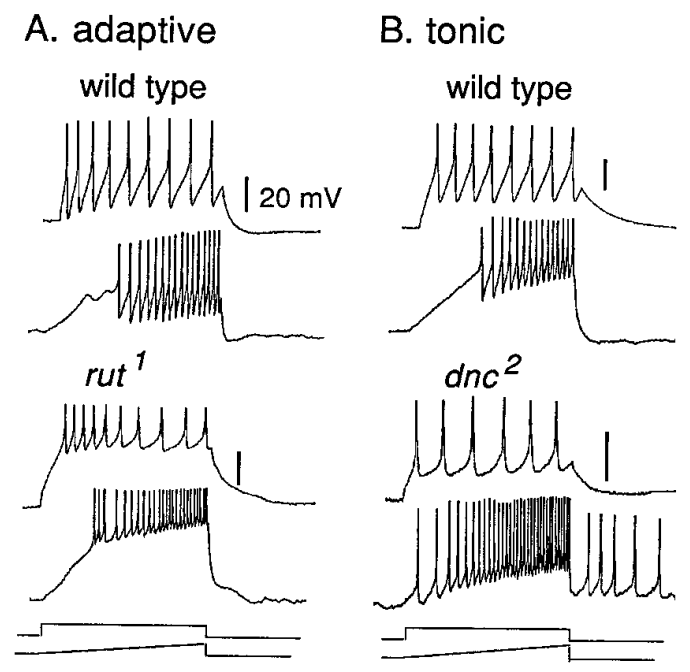

\section{D. adaptive}


\section{C. delayed}
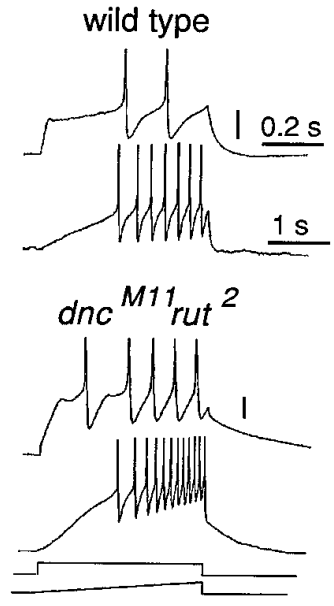

E. tonic
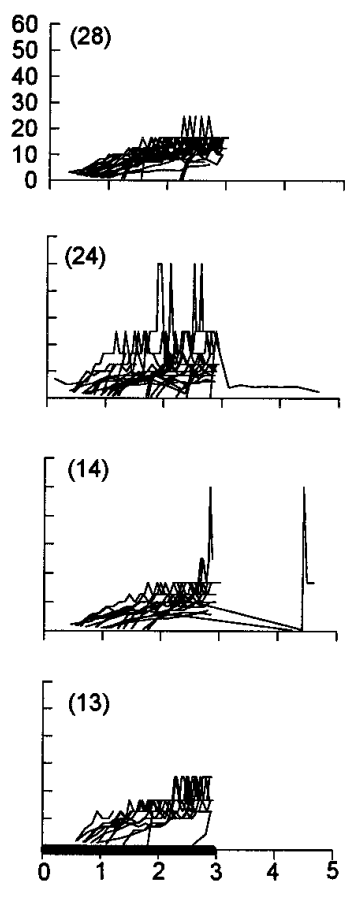
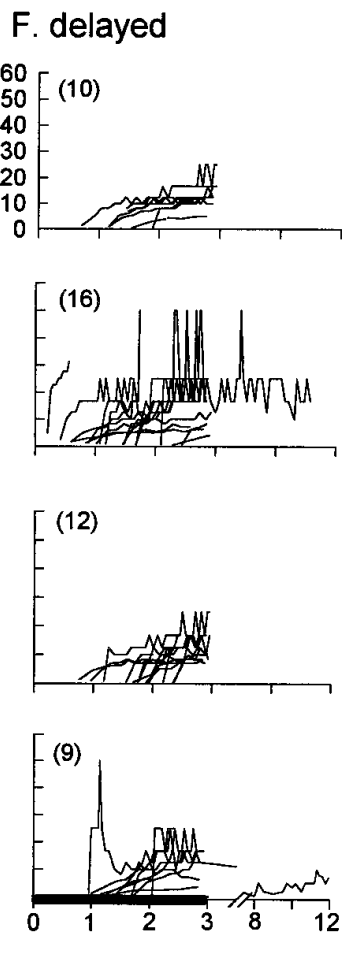

Figure 9. Firing of neurons in adaptive $(A)$, tonic $(B)$, and delayed $(C)$ categories in response to 600 msec step current injections (top traces) and $3 \mathrm{sec}$ ramp stimuli (bottom traces). Examples from wildtype (top panel) and mutant (bottom panel) neurons are shown. Note different time and voltage scales. $D-F$, Instantaneous frequencies of spike trains evoked after the onset of the $3 \mathrm{sec}$ ramp in neurons of tonic $(D)$, adaptive $(E)$, and delayed $(F)$ categories. Neurons of each genotype are grouped for comparison (see Fig. 6 for details of the continuation for the instantaneous frequency plots). Note sustained firing after the cessation of ramp (bars beneath the time axis) in some mutant neurons. establishes the functional categories of Drosophila neurons according to distinct firing patterns.

An understanding of the functional diversity of neurons is required for a broad biological framework for studying the effects of defined molecular lesions on single neurons in Drosophila mutants with specific behavioral defects. Four distinct types of firing patterns, tonic, adaptive, delayed, and interrupted, were classified according to the instantaneous frequency and first spike latency (Fig. 2). We provided different lines of evidence suggesting that distinct firing patterns of neurons are associated with differential expression of the transient, 4-AP-sensitive, and delayed, TEA-sensitive $\mathrm{K}^{+}$currents (Figs. 3, 4). Significantly, the categories of spike activities in Drosophila neurons show striking parallels to those described in other species, including mammals (Llinás, 1988; Connors and Gutnick, 1990).

Despite the opposite effects on cAMP metabolism, $d n c$ and rut mutations (Byers et al., 1981; Livingstone et al., 1984) lead to superficially similar defects in the various developmental, physiological, and behavioral phenotypes. These include poor learning performance (Tully and Quinn, 1985; Wustmann et al., 1996), abnormal habituation of a cleaning reflex (Corfas and Dudai, 1989) and an escape circuit (Engel and Wu, 1996) in adult flies, altered synaptic transmission at the larval neuromuscular junction (Zhong and Wu, 1991), reduced growth cone motility in cultured larval CNS neurons (Kim and Wu, 1996), and disrupted frequency coding and hyperexcitability in cultured giant neurons reported here. Nevertheless, striking differences in the counterbalancing effects of $d n c$ and rut have been observed in morphological and functional assays. Abnormalities in both branching patterns of nerve terminals (Zhong and Wu, 1992) and growth cone motility (Kim and $\mathrm{Wu}, 1996$ ) could be restored in dnc rut double mutants. In contrast, severe behavioral and physiological alterations still 
A. adaptive

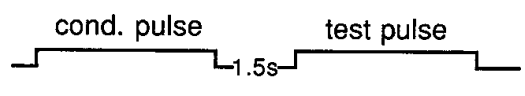

wild type

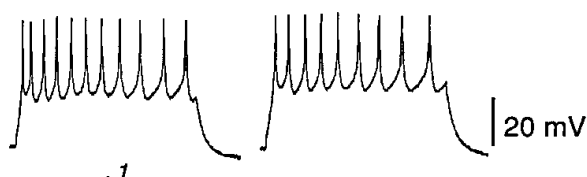

rut $^{1}$
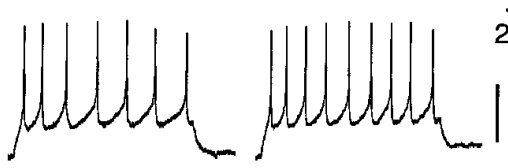

$200 \mathrm{~ms}$

\section{B. delayed}

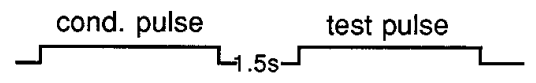

wild type

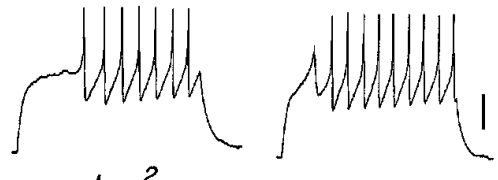

$d n c^{2}$

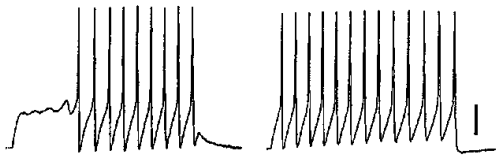

Figure 10. Changes in neuronal firing rate after a prolonged $(2 \mathrm{sec})$ preconditioning pulse. $A, B$, Examples of spike trains observed from wild-type (top traces) and mutant (bottom traces) neurons of the adaptive $(A)$ and delayed $(B)$ categories. Spikes elicited by the conditioning current injection (left panels) are compared with spikes in response to the test pulse of identical duration and amplitude (right panels), with the interpulse interval of $0.5-1$ sec. $C-E$, Cumulative change of spike numbers from different categories of neurons. For each neuron, the spike counts during the conditioning $\left(n_{\text {cond. }}\right)$ and the test $\left(n_{\text {test }}\right)$ pulse are compared at each time point (the bin size is $0.2 \mathrm{sec}$ ) after the onset of stimulation. The cumulative difference in the spike counts between the two spike trains $\left(n_{\text {test }}-n_{\text {cond }}\right)$ is plotted against time. Note the different influence of the conditioning pulse on the firing rate in tonic $(C)$, adaptive $(D)$, and delayed $(E)$ wild-type neurons and the lack of such trends in mutant neurons of some categories.

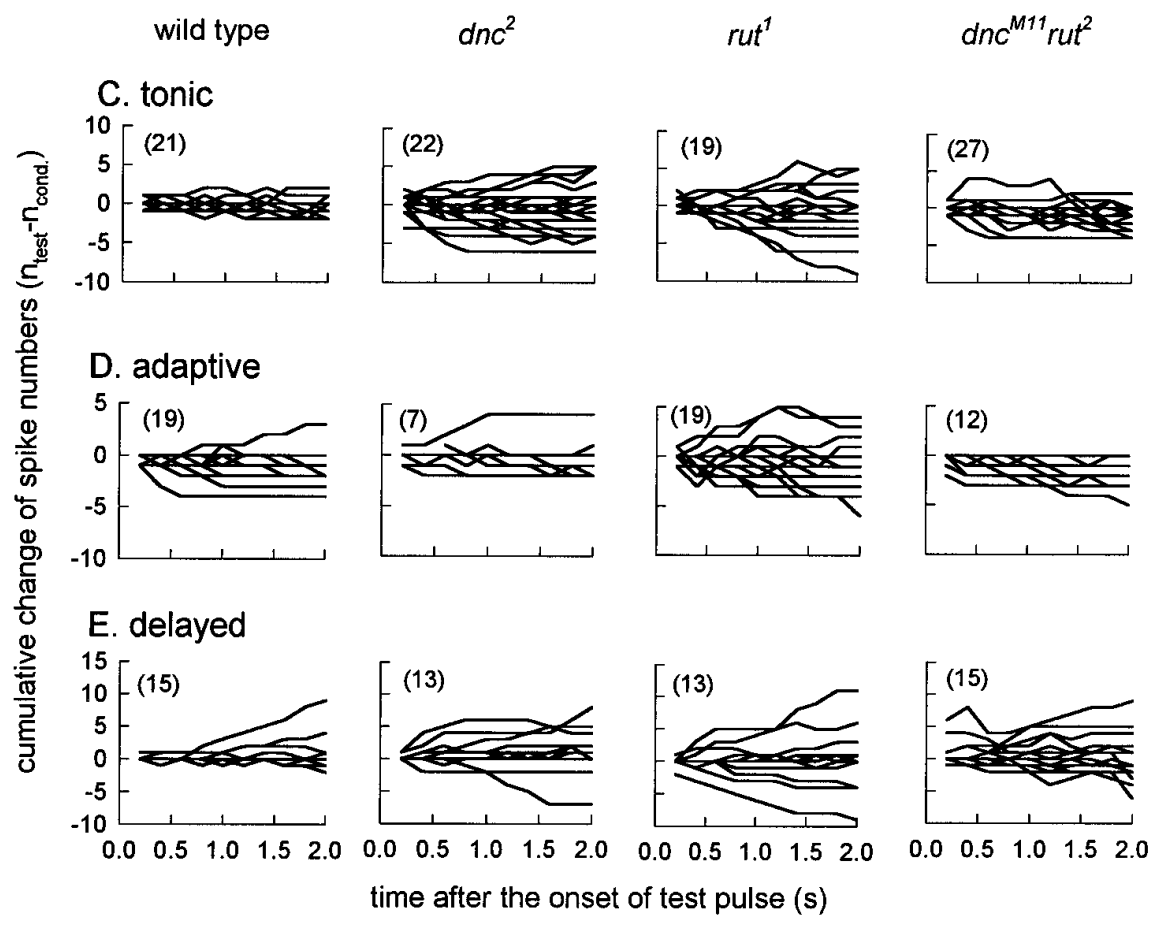

remain in double mutants, including poor learning performance (Livingstone et al., 1984) and more severe defects in habituation of an escape circuit (Engel and Wu, 1996), and deranged firing patterns in cultured neurons (this report), suggesting that $d n c$ could not counteract the rut defects in these functions.

Several known cellular mechanisms must be considered in future research into the reasons why $d n c$ and rut mutations show different abilities to counteract in different phenotypes. First, it is possible that the enzymes phosphodiesterase (PDE) and adenylyl cyclase (AC), which are encoded by these two genes (Chen et al., 1986; Levin et al., 1992), are segregated and localized to different subcellular compartments (Nighorn et al., 1991; Han et al., 1992) associated with different downstream targets. Alternatively, the dynamic process of the cAMP cascade (Levine et al., 1994), rather than the average levels of cAMP, might be crucial for neural plasticity. Thus, the striking alterations of neuronal activity that remained in $d n c$ rut double mutants may indicate that an intact spatial and temporal organization of the pathway is required to conduct the regulation and modulation of ion channels in the neuron.
Similar to $d n c$ and rut, ion channel mutations such as Sh (Shaker) and eag (ether á go-go), which affect voltage-dependent $\mathrm{K}^{+}$channels, and nap ${ }^{\text {ts }}$ (no action potential-ts), which suppresses expression of voltage-dependent $\mathrm{Na}^{+}$channels $(\mathrm{Wu}$ and Ganetzky, 1992), have been demonstrated to cause short-term memory deficiency in an odor-associative learning paradigm (Cowan and Siegel, 1984, 1986). This may not be surprising, because the modulation of various $\mathrm{K}^{+}$currents in larval muscle has been shown to be altered in $d n c$ or rut mutants (Zhong and $\mathrm{Wu}, 1993)$. Furthermore, our voltage-clamp results indicate that the density of $\mathrm{K}^{+}$currents is significantly reduced in rut and $d n c$ rut (Fig. 11), suggesting that some mutant phenotypes may be caused by perturbed modulation of $\mathrm{K}^{+}$current. It should be noted that other ion channels, in addition to voltage-activated $\mathrm{K}^{+}$channels, are likely to be affected in $d n c$ and rut (M.-L. Zhao and C.-F. $\mathrm{Wu}$, unpublished observation). The feasibility of performing both current- and voltage-clamp recordings on the same cells in the giant neuron culture system, combined with available vital markers for identification of neuronal types (enhancer-trap detection: Wright and Zhong, 1995; green fluorescent protein technique: 
A

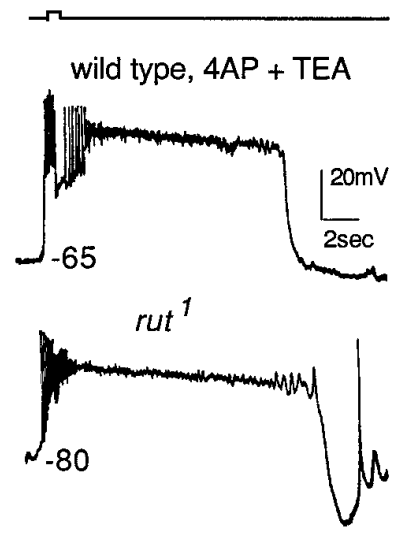

B

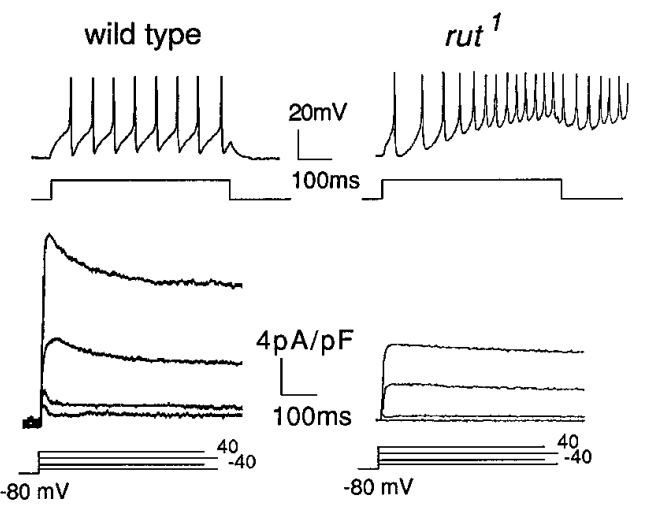

C

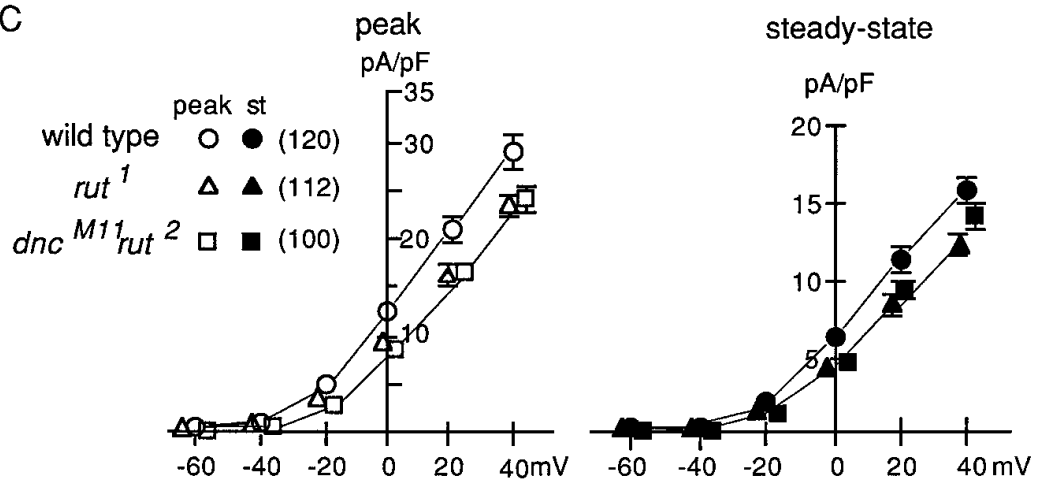

Figure 11. Reduced density of voltage-dependent $\mathrm{K}^{+}$currents in rut and dnc rut. A, Phenocopy of the long-lasting firing seen in some rut neurons (bottom) by a wild-type neuron treated with both $2 \mathrm{~mm} 4-\mathrm{AP}$ and $10 \mathrm{~mm}$ TEA (top). B, Correlation of firing mode and the underlying $\mathrm{K}^{+}$ currents in the same neurons of wild-type control and the $r^{r u t}{ }^{1}$ mutant. Suppression of voltage-activated $\mathrm{K}^{+}$currents is evident. $C$, Current-voltage relations showing density of $\mathrm{K}^{+}$currents determined at the peak and steady-state from populations of wild-type and mutant neurons. Most of the cells were not characterized for their firing patterns. Data points indicate mean \pm SEM, with the number of cells examined shown in parentheses.

Yeh et al., 1995), should enable additional studies on the various ionic currents that underlie $d n c$ and rut phenotypes and that contribute to neuronal plasticity.

\section{Altered frequency coding and responses to previous conditioning in memory mutants}

Many lines of evidence from both invertebrates and vertebrates have demonstrated that neuronal spike activity plays an important role in synaptic plasticity in vivo (Lnenicka et al., 1986; Budnick et al., 1990; Cline, 1991). In addition, neuronal electrical properties can exhibit considerable plasticity, as has been shown in the thalamus of mammalian brain (McCormick, 1992) and the somatogastric ganglia of lobster (Turrigiano et al., 1994, 1995). Various firing patterns found in Drosophila neurons are directly comparable with the heterogeneous firing properties in mammalian cortical neurons (Conners and Gutnick, 1990; Kawaguchi, 1995) and in identified invertebrate neurons (Byrne, 1980; Getting, 1983; Johansen and Kleinhaus, 1990). Therefore, modulation of neuronal firing patterns in Drosophila should be characterized in detail to explore further the genetic control of cellular mechanisms underlying neuroplasticity.

In response to suprathreshold step current injections, wild-type neurons of different categories follow a defined temporal pattern in firing frequency, and each operates within a restricted frequency range (Figs. 2, 3; Table 1). In contrast, erratic firing patterns in subsets of $d n c$ and rut mutant neurons deviate from a clear scheme of frequency coding for each cell category (Figs. 5, 6). Some details of the abnormalities are noteworthy. First, the periodic bursting activity of single mutant neurons reached an instantaneous spike frequency as high as $120 \mathrm{~Hz}$ (Fig. 6), whereas the maximum instantaneous frequency seldom approached $30 \mathrm{~Hz}$ in wild-type controls. Such bursting activities apparently occurred more frequently in tonic and delayed neurons than in adaptive neurons. Second, unlike wild-type neurons that returned to quiescence at the termination of stimulation (Figs. 5, 6), some mutant neurons frequently generated prolonged firing activities outlasting current steps for seconds (Fig. 6, indicated by arrows). These long-lasting potentials seemed to be more frequent in neurons of rut than those of $d n c$. Third, extreme cases of abnormal patterns of regenerative potentials were found in subpopulations of mutant neurons that do not fall into the four categories in response to step current injections (Fig. 7).

Additional subtleties of mutational effects on neuronal excitability were revealed with stimulation paradigms (Fig. 8) involving preconditioning, such as a progressive increment of stimulation strength in the ramp or long-duration depolarization in the twinpulse protocol. In general, mutant neurons displayed in these two paradigms showed considerably greater variability than wild-type controls (Figs. 9, 10). Moreover, the overall trend found in each category of wild-type controls with a twin-pulse paradigm became blurred in $d n c$ and rut mutant neurons (Fig. 10). So far, these paradigms have examined only short-term plasticity in neuronal excitability. The long-term effects of conditioning by prolonged previous activity on firing patterns in Drosophila neurons must await further investigation.

Synchronous activities and oscillations at characteristic firing frequencies in neuronal populations are thought to be important for the proper functioning of isolated neuronal networks of the rat hippocampus and neocortex (Buzsáki et al., 1992; Whittington et al., 1995). Recently, theoretical analysis and computational modeling proposed that multiple short-term memory events could be represented by oscillatory activities in a network, with each memory event stored at a different high-frequency subcycle imbedded 
in a low-frequency oscillation (Lisman and Idiart, 1995). Progress made in insects revealed that the frequency of field potential oscillations in the mushroom bodies of the locust is odordependent (Laurent and Naraghi, 1994), with processing of different features of olfactory information distributed among neural subassemblies (Laurent and Davidowitz, 1994). The observed aberrant spontaneous activity, disrupted frequency coding, and abnormal modulation by previous conditioning in $d n c$ and rut neurons of Drosophila might present problems in the stability of neural circuits and the reliability of information processing (Sanes and Constantine-Paton, 1983; Getting, 1989), causing poor performance in certain learning tasks in mutants. Our results thus lend strong support for the notion that in addition to the well established synaptic mechanisms, modulation of neuronal excitability represents a potentially important cellular mechanism for learning and memory processes.

\section{REFERENCES}

Bailey CH, Kandel ER (1993) Structural changes accompanying memory storage. Annu Rev Physiol 55:397-426.

Baker K, Salkoff L (1990) The Drosophila Shaker gene codes for a distinctive $\mathrm{K}^{+}$current in a subset of neurons. Neuron 2:129-140.

Bekkers JM, Stevens CF (1991) Excitatory and inhibitory autaptic currents in isolated hippocampal neurons maintained in cell culture. Proc Natl Acad Sci USA 88:7834-7838.

Bliss TVP, Collingridge GL (1993) A synaptic model of memory: longterm potentiation in the hippocampus. Nature 361:31-39.

Budnick V, Zhong Y, Wu C-F (1990) Morphological plasticity of motor axons in Drosophila mutants with altered excitability. J Neurosci 10:3754-3768.

Buzsáki G, Horváth Z, Urioste R, Hetke J, Wise K (1992) Highfrequency network oscillation in the hippocampus. Science 256:1025-1027.

Byerly L, Leung HT (1988) Ionic currents of Drosophila neurons in embryonic cultures. J Neurosci 8:4379-4393.

Byers D, Davis RL, Kiger Jr JA (1981) Defect in cyclic AMP phosphodiesterase due to the dunce mutation of learning in Drosophila melanogaster. Nature 289:79-81.

Byrne JH (1980) Quantitative aspects of ionic conductance mechanisms contributing to firing pattern of motor cells mediating inking behavior in Aplysia calicornica. J Neurophysiol 43:651-668.

Chen CN, Denome S, Davis RL (1986) Molecular analysis of cDNA clones and the corresponding genomic coding sequences of the Drosophila dunce ${ }^{+}$gene, the structural gene for cAMP phosphodiesterase. Proc Natl Acad Sci USA 83:9313-9317.

Cline HT (1991) Activity-dependent plasticity in the visual systems of frogs and fish. Trends Neurosci 14:104-111.

Connors BW, Gutnick MJ (1990) Intrinsic firing patterns of diverse neocortical neurons. Trends Neurosci 13:99-104.

Corfas G, Dudai Y (1989) Habituation and dishabituation of a cleaning reflex in normal and mutant Drosophila. J Neurosci 9:56-62.

Cowan TM, Siegel RW (1984) Mutational and pharmacological alterations of neuronal membrane function disrupt conditioning in Drosophila. J Neurogenet 1:333-344.

Cowan TM, Siegel RW (1986) Drosophila mutations that alter ionic condition disrupt acquisition and retention of a conditioned odor avoidance response. J Neurogenet 3:187-201.

Davis RL (1996) Physiology and biochemistry of Drosophila learning mutants. Physiol Rev 76:299-317.

DeZazzo J, Tully T (1995) Dissection of memory formation: from behavioral pharmacology to molecular genetics. Trends Neurosci 18:212-218.

Dudai Y, Jan Y-N, Byers D, Quinn WG, Benzer S (1976) dunce, a mutant of Drosophila deficient in learning. Proc Natl Acad Sci USA 73:1686-1688.

Engel JE, Wu C-F (1996) Alteration of non-associative conditioning of an identified escape circuit in Drosophila memory mutants. J Neurosci 16:3486-3499.

Frey U, Huang Y-Y, Kandel ER (1993) Effects of cAMP stimulate a late stage of LTP in hippocampal CA1 neurons. Science 260:1661-1664.

Getting PA (1983) Mechanisms of pattern generation underlying swimming in Tritonia. III. Intrinsic and synaptic mechanisms for delayed excitation. J Neurophysiol 49:1036-1050.
Getting PA (1989) Emerging principles governing the operation of neural networks. Annu Rev Neurosci 12:185-204.

Han P-L, Levin LR, Reed RR, Davis RL (1992) Preferential expression of the Drosophila rutabaga gene in mushroom bodies, neural centers for learning in insects. Neuron 9:619-627.

Harris-Warrick RM, Marder E (1991) Modulation of neural networks for behavior. Annu Rev Neurosci 14:39-57.

Hille B (1992) Ion channels of excitable membrane. Sunderland, MA: Sinauer.

Huff R, Furst A, Mahowald AP (1989) Drosophila embryonic neuroblasts in culture: autonomous differentiation of specific neurotransmitters. Dev Biol 134:146-157.

Huang Y-Y, Li XC, Kandel ER (1994) cAMP contributes to mossy fiber LTP by initiating both a covalently mediated early phase and macromolecular synthesis-dependent late phase. Cell 79:69-79.

Ikeda K, Kaplan WD (1974) Neurophysiological genetics in Drosophila melanogaster. Am Zool 14:1055-1066.

Johansen J, Kleinhaus AL (1990) Ionic conductances in two types of sensory neurons in the leech. Comp Biochem Physiol 97:577-582.

Kaczmarek LK, Kauer JA (1983) Calcium entry causes a prolonged refractory period in peptidergic neurons of Aplysia. J Neurosci 11:2230-2239.

Kandel ER, Schwartz JH (1982) Molecular biology of learning: modulation of transmitter release. Science 218:433-443.

Kawaguchi Y (1995) Physiological subgroups of nonpyramidal cells with specific morphological characteristics in layer II/III of rat frontal cortex. J Neurosci 15:2638-2655.

Kim Y-T, Wu C-F (1996) Reduced growth cone motility in cultured neurons from Drosophila memory mutants with a defective cyclic AMP cascade. J Neurosci 16:5593-5602.

Laurent G, Davidowitz H (1994) Encoding of olfactory information with oscillating neural assemblies. Science 265:1872-1875.

Laurent G, Naraghi M (1994) Odorant-induced oscillations in the mushroom bodies of the locust. J Neurosci 14:2993-3004.

Levin LR, Han P-L, Hwang PM, Feinstein PG, Davis RL, Reed RR (1992) The Drosophila learning and memory gene rutabaga encodes a $\mathrm{Ca}^{2+} /$ calmodulin-responsive adenylate cyclase. Cell 68:479-489.

Levine JD, Casey CI, Kalderon DD, Jackson FR (1994) Altered circadian pacemaker functions and cyclic AMP rhythms in the Drosophila learning mutant dunce. Neuron 13:967-974.

Levitan IB (1988) Modulation of ion channels in neurons and other cells. Annu Rev Neurosci 11:119-136.

Li M, West JW, Lai Y, Scheuer T, Catterall WA (1992) Functional modulation of brain sodium channels by cAMP-dependent phosphorylation. Neuron 8:1151-1159.

Livingstone MS, Sziber PP, Quinn WG (1984) Loss of calcium/calmodulin responsiveness in adenylate cyclase of rutabaga, a Drosophila learning mutant. Cell 37:205-215.

Lisman JE, Idiart MAP (1995) Storage of $7 \pm 2$ short-term memories in oscillatory subcycles. Science 267:1512-1515.

Llinás RR (1988) The intrinsic electrophysiological properties of mammalian neurons: insights into central nervous system function. Science 242:1654-1664.

Lnenicka GA, Atwood HL, Marin L (1986) Morphological transformation of synaptic terminals of a phasic motoneuron by long-term tonic stimulation. J Neurosci 6:2252-2258.

Marcus EA, Carew TJ (1991) Development and modulation of endogenous bursting in identified neuron R15 of juvenile Aplysia. J Neurobiol 22:418-429.

McCormick DA (1992) Cellular mechanisms underlying cholinergic and noradrenergic modulation of neuronal firing mode in the cat and guinea pig dorsal lateral geniculate nucleus. J Neurosci 12:278-289.

Nighorn A, Healy MJ, Davis RL (1991) The cyclic AMP phosphodiesterase encoded by the Drosophila dunce gene is concentrated in the mushroom body neuropil. Neuron 6:455-467.

O'Dowd DK (1995) Voltage-gated currents and firing properties of embryonic Drosophila neurons grown in a chemically defined medium. J Neurobiol 27:113-126.

Rogawski MA (1985) The A-current: how ubiquitous a feature of excitable cells is it? Trends Neurosci 8:214-219.

Saito M, Wu C-F (1991) Expression of ion channels and mutational effects in "giant" Drosophila neurons differentiated from cell divisionarrested embryonic neuroblasts. J Neurosci 11:2135-2150.

Saito M, Wu C-F (1993) Ionic channels in cultured Drosophila neurons. 
In: Comparative molecular neurobiology (Pichon Y, ed), pp 366-389. Basel: Birkhauser.

Salkoff L (1983) Genetic and voltage clamp analysis of a Drosophila potassium channel. Cold Spring Harbor Symp Quant Biol 48:221-231.

Sanes DH, Constantine-Paton M (1983) Altered activity patterns during development reduce neural tuning. Science 221:1183-1185.

Singh S, Wu C-F (1989) Complete separation of four potassium currents in Drosophila. Neuron 2:1325-1329.

Solc CK, Aldrich RW (1988) Voltage-gated potassium channels in larval CNS neurons of Drosophila. J Neurosci 8:2556-2570.

Tanouye MA, Ferrus A, Fujita SC (1981) Abnormal action potentials associated with the Shaker complex locus of Drosophila. Proc Natl Acad Sci USA 78:6548-6552.

Tully T, Quinn WA (1985) Classical conditioning and retention in normal and mutant Drosophila melanogaster. J Comp Physiol 157:263-277.

Turrigiano G, Abbott LF, Marder E (1994) Activity-dependent changes in the intrinsic properties of cultured neurons. Science 264:974-977.

Turrigiano G, LeMasson G, Marder E (1995) Selective regulation of current densities underlies spontaneous changes in the activity of cultured neurons. J Neurosci 15:3640-3652.

Wang JW, Wu C-F (1996) In vivo functional role of the Drosophila Hyperkinetic $\beta$ subunit in gating and inactivation of Shaker $\mathrm{K}^{+}$channels. Biophys J 71:3167-3176.

Weisskopf MG, Castillo PE, Zalutsky RA, Nicoll RA (1994) Mediation of hippocampal mossy fiber long-term potentiation by cyclic AMP. Science 265:1878-1882.

Whittington MA, Traub RD, Jefferys JGR (1995) Synchronized oscillations in interneuron networks driven by metabotropic glutamate receptor activation. Nature 373:612-615.

Wright NJD, Zhong Y (1995) Characterization of $\mathrm{K}^{+}$currents and the cAMP-dependent modulation in cultured Drosophila mushroom body neurons identified by LacZ expression. J Neurosci 15:1025-1034.

Wu C-F, Ganetzky B (1992) Neurogenetic studies of ion channels in Drosophila. In: Ion channels, Vol 3 (Narahashi T, ed), pp 261-314. New York: Plenum.

Wu C-F, Haugland FN (1985) Voltage-clamp analysis of membrane currents in larval muscle fibers of Drosophila: alterations of potassium currents in Shaker mutants. J Neurosci 5:2626-2640.

Wu C-F, Sakai K, Saito M, Hotta Y (1990) Giant Drosophila neurons differentiated from cytokinesis-arrested embryonic neuroblasts. J Neurobiol 21:499-507.

Wustmann G, Rein K, Wolf R, Heisenberg M (1996) A new paradigm for operant conditioning of Drosophila melanogaster. J Comp Physiol [A] 179:429-436.

Yeh E, Gustafson K, Boulianne G (1995) Green fluorescent protein as a vital marker and reporter of gene expression in Drosophila. Proc Natl Acad Sci USA 92:7036-7040.

Zhao M-L, Wu C-F (1994) Altered spike activity in cultured "giant" neurons derived from dunce and rutabaga mutants of Drosophila. Soc Neurosci Abstr 20:803.

Zhao M-L, Sable E, Iverson L, Wu C-F (1995) Functional expression of Shaker $\mathrm{K}^{+}$channels in cultured Drosophila "giant" neurons derived from $S h$ cDNA transformants: distinct properties, distribution, and turnover. J Neurosci 15:1406-1418.

Zhong Y, Wu C-F (1991) Altered synaptic plasticity in Drosophila memory mutants with a defective cyclic AMP cascade. Science 251:198-201.

Zhong Y, Wu C-F (1993) Differential modulation of potassium currents by cAMP and its long-term and short-term effects: dunce and rutabaga mutants of Drosophila. J Neurogenet 9:15-27.

Zhong Y, Budnik V, Wu C-F (1992) Synaptic plasticity in Drosophila memory and hyperexcitable mutants: role of cAMP cascade. J Neurosci 12:644-651. 